

\title{
On the use of parataxonomy in biodiversity monitoring: a case study on wild flora
}

Jean-Claude Abadie, Camila Andrade, Nathalie Machon, Emmanuelle Porcher

\section{To cite this version:}

Jean-Claude Abadie, Camila Andrade, Nathalie Machon, Emmanuelle Porcher. On the use of parataxonomy in biodiversity monitoring: a case study on wild flora. Biodiversity and Conservation, 2008, 17 (14), pp.3485-3500. 10.1007/s10531-008-9354-z . halsde-00386008

\section{HAL Id: halsde-00386008 https://hal.science/halsde-00386008}

Submitted on 12 Aug 2019

HAL is a multi-disciplinary open access archive for the deposit and dissemination of scientific research documents, whether they are published or not. The documents may come from teaching and research institutions in France or abroad, or from public or private research centers.
L'archive ouverte pluridisciplinaire HAL, est destinée au dépôt et à la diffusion de documents scientifiques de niveau recherche, publiés ou non, émanant des établissements d'enseignement et de recherche français ou étrangers, des laboratoires publics ou privés. 


\section{On the use of parataxonomy in biodiversity}

\section{monitoring: a case study on wild flora}

Jean-Claude ABADIE ${ }^{1,2 *}$, Camila ANDRADE ${ }^{1}$, Nathalie MACHON ${ }^{1,2}$, Emmanuelle PORCHER ${ }^{1,2}$

${ }^{1}$ UMR5173 Conservation des espèces, restauration et suivi des populations et USM 2699 Inventaire et suivi de la biodiversité, Conservatoire Botanique National du Bassin Parisien, Muséum National d'Histoire Naturelle, 61 rue Buffon, 75005 PARIS 2 USM 2699 Inventaire et suivi de la biodiversité, Conservatoire Botanique National du Bassin Parisien, Muséum National d'Histoire Naturelle, 61 rue Buffon, 75005 PARIS

*Corresponding author; E-mail abadie@mnhn.fr

\section{Abstract:}

Monitoring programs that assess species richness and turnover are now regarded as essential to document biodiversity loss worldwide. Implementation of such programs is impeded by a general decrease in the number of skilled naturalists. Here we studied how morphotypes, instead of species, might be used by unskilled participants (referred to as "volunteers") to survey common plant communities. Our main questions were: (1) Can morphotypes be used as a robust estimator of species richness ( $\alpha$-diversity) and assemblage turnover ( $\beta$-diversity)? and (2) What is the robustness (reproducibility and repeatability) of such methods? Double inventories 
were performed on 150 plots in arable field margins, one by a non-expert using morphotypes, the other by a taxonomist using species. To test the robustness of morphotype identification among participants, twenty additional plots were surveyed by eight volunteers using the same protocol. We showed that (1) the number of morphotypes identified by unskilled volunteers in a plot was always strongly correlated with species richness. (2) Morphotypes were sensitive to differences among habitats but were less accurate than species to detect these differences. (3) Morphotype identification varied significantly within and between volunteers. Due to this lack of repeatability and reproducibility, parataxonomy cannot be considered a good surrogate for taxonomy. Nevertheless, assuming that morphotypes are identified with standardised methods, and that results are used only to evaluate gross species richness but not species turnover, parataxonomy might be a valuable tool for rapid biodiversity assessment of common wild flora.

\section{Keywords:}

Citizen science; Inventory; Monitoring; Morphotypes; Parataxonomy; Sampling bias; Taxonomy; Wider countryside

\section{Introduction}

Over the last couple of centuries, Europe has been submitted to strong human pressures (Stoate et al. 2001), which have resulted mostly in agricultural intensification (Tscharntke et al. 2005), landscape fragmentation (Fischer and Lindenmayer 2007) and increased urbanization (McKinney 2006). As a consequence, large regions have become poor-quality habitats for wildlife, and a lot of species considered common in the beginning of the twentieth century are now 
experiencing severe declines, or even becoming scarce (Smart et al. 2005; Rich 2006). These areas and the ecological communities they support, usually referred to as "the wider landscape matrix" (e.g. wider countryside, urban and suburban areas), are subsequently less attractive for conservationists and often remain excluded from conservation planning (Gaston and Fuller 2007). In this context, the need for largescale, long-term monitoring programs embracing non-protected areas is now widely recognized (Yoccoz et al. 2001; Pereira and Cooper 2006; Teder et al. 2007). Such programs however require a large number of observation points, and their implementation is often hindered by the lack of species identification skills, arising from a decrease in the number of taxonomists, both professionals (Whitehead 1990; Noss 1996; Rivas 1997) and amateurs (Hopkins and Freckleton 2002). These difficulties are less severe in the wider countryside, supporting common species, which are usually familiar to naturalists. These species remain nonetheless unattractive for the majority of naturalists, who generally prefer atypical ecosystems and/or rare species, despite the fact that the wider countryside (e.g. agricultural landscapes, covering vast areas in Europe) now experiences substantial species decline (Perrings et al. 2006).

With the rise of "citizen science" (Trumbull et al. 2000; Lundmark 2003; Andrianandrasana et al. 2005), where non-professional observers are involved in monitoring programs (Penrose and Call 1995; Fore et al. 2001; Engel and Voshell 2002; Pilgrim and Hutchinson 2003; Stewart et al. 2006), volunteering might be an alternative to develop the monitoring of common species. The involvement of volunteers has been shown to facilitate data collection for taxonomic studies (e.g. specimens, Janzen 2004) or environmental monitoring (e.g. presence of a species or environmental features, repeated over time Holck 2007). However, the participation 
of volunteers in biodiversity studies also generates scepticism among the scientific community about the reliability of data collected by inexperienced observers (FosterSmith and Evans 2003). Some ecologists argue that these approaches may compromise data accuracy and increase biases beyond acceptable levels, in comparison with data collected by professional biologists (Firehock and West 1995). In addition, taxonomists highlight that the involvement of non professional observers might act as competition and therefore be an impediment to the maintenance and renewal of the taxonomy community (Goldstein 1997). This however depends on the actual skills of volunteers. Many so-called 'citizen science' programs (including breeding bird surveys Julliard and Jiguet 2002b; Newson et al. 2005), involve amateur naturalists whose skills can generally not be questioned. Other approaches, which we investigate here, may involve non-naturalist volunteers (Evans et al. 2005); in this case, alternative methods for species identification (paratoxonomy) are required to compensate for their virtually complete lack of taxonomic knowledge (Fore et al. 2001).

Parataxonomy consists of using morphotypes, also referred to as parataxonomic units (Krell 2004) or recognizable taxonomic units (Cranston and Hillman 1992; Oliver and Beattie 1993), as a surrogate for taxonomic species. Paratoxonomic methods have been used in tropical ecosystems, generally characterized by high species richness, including numerous undiscovered or undescribed species, where there is a chronic lack of on-site trained taxonomists (Gaston and May 1992; Basset et al. 2004). These methods are usually a preliminary step towards species determination (Wheeler 1995): parataxonomists, also called biodiversity technicians (Longino and Colwell 1997; Janzen 2004), sort voucher specimens on the basis of morphological similarities. Voucher specimens are then 
generally determined at the species level by professional taxonomists, but the morphotypes may sometimes be considered to provide sufficient information, especially in invertebrate groups, in which case species are not determined (Oliver and Beattie 1993; Oliver and Beattie 1996b; Oliver and Beattie 1996a; Oliver et al. 2000; Derraik et al. 2002). So far, parataxonomic approaches have been used for taxonomic groups with significant identification difficulties, essentially invertebrates (Pik et al. 1999; Ward and Larivière 2004), such as arthropods (Bolger et al. 2000; Kerr et al. 2000) or Rotifera (Nielsen et al. 1998), and in one instance bryophytes (Oliver and Beattie 1993). With the development of "citizen science", parataxonomy could be an effective way to boost the number of biodiversity surveyors, particularly in common ecosystems supporting well-known species, where it could prove useful to counterbalance the lack of trained taxonomists. To our knowledge, such approach has never been applied to large-scale monitoring.

The goal of this paper is to evaluate the potential of parataxonomy for monitoring plant communities in the wider countryside. To achieve this, we set up a simple parataxonomic method, requiring almost no a priori knowledge of botany and using a standardized protocol based on simplified determination keys. We then performed a comparison of taxonomic and parataxonomic methods in an agricultural landscape to investigate the following questions: (1) Can morphotypes be used to generate robust estimators of species richness and turnover? (2) Does this method meet two important criteria of scientific method, i.e. reproducibility and repeatability (Cassey and Blackburn 2006)?

\section{Material and Methods}


Field site and sampling protocol

We conducted field sampling in the Parisian region, a highly agricultural region, according to the following protocols. For all botanical inventories nomenclature follows (Kerguélen 2003).

\section{Botanist versus volunteer experiment}

Fifteen fields were selected among the 1000 sites of the "Biovigilance" Biodiversity Observatory Network (Fried et al. 2005) and sampled in spring 2006 following a standardized protocol used to monitor the abundance of flora in arable field margins. Ten $1 \mathrm{~m}^{2}$ rectangular plots were placed evenly on the margins of each field (yielding a total of 150 plots), so that a wide variety of margin types were represented: grass strip (16 plots), footpath (79 plots), fallow land (15 plots), road side (15 plots), and wood edge (25 plots). For this experiment two observers were involved in surveying each plot: a trained botanist, referred to as "botanist", and a student with no experience in plant determination, referred to as "volunteer". In each plot, the botanist inventoried all vascular plant species as exhaustively as possible, while the volunteer independently estimated the number and identity of morphotypes, using a simplified method (see below).

\section{Multiple-volunteer experiment}

To examine the variation among individual observers (both within botanists and within volunteers) and the reproducibility of parataxonomic methods when 
several observers are involved, we repeated the procedure above with eight volunteers and three botanists in a subsample of 20 plots, encompassing four different types of field margin: grass strip, fallow land, hedge and wood edge (5 plots per margin type). Volunteers included people of various academic levels, with a background in biology, but with no experience in field work and plant determination, so that we can assume that their qualification for parataxonomy is similar to what we might expect of non naturalists following similar protocols. During the field session, all botanists and volunteers first performed inventories independently, to avoid identification bias. Each morphotype found by a volunteer was then individually examined by a botanist and assigned to one or more species.

\section{Defining plant morphotypes}

The morphotype identification process used by volunteers was based on identification keys, but differs in that it is not based only on dichotomous choices. Plants were first separated into forbs and graminoids, and then characterized via a number of easily observable characters. For each character, a list of the different character states, encoded by a letter or a number, was provided to the observers (Figure 1). Each morphotype was thus defined by a code corresponding to a combination of character states. For forbs, six characters were considered sufficient to discriminate the various plant morphologies: (1) leaf shape (24 states, see Figure 1), (2) growth form (two states, rosette and erected), leaf arrangement (three states, alternate, opposite, whorled), pubescence (two states, present or absent), and flower colour (if present, four states, white, yellow, red or blue). For graminoids, only leaf width was considered, with three levels (thin, intermediate and wide). A strict 
minimum of scientific terms was used and drawings were provided to illustrate or even replace lists of scientific terms (e.g. leaf shape).

\section{Data analysis}

In the following we define $\alpha$-diversity as within habitat diversity and $\beta$-diversity as the degree of change in species diversity among habitats (Whittaker 1972). Data analyses were similar for both experiments. We first calculated the "gross" accuracy, i.e. the number of taxonomically identified species minus the number of morphotypes, divided by the number of species (Krell 2004). The efficiency in estimation of $\alpha$ diversity using morphotypes was assessed by considering the relationship between species richness and morphotype richness per plot, using Spearman correlations. The congruence between $\beta$-diversity measured with species vs. morphotypes was investigated indirectly by comparing dissimilarity among replicates, using the semimetric Bray-Curtis distance (Bray and Curtis 1957), generally considered one of the best measures of community dissimilarity (Clarke 1993). As it was not possible to monitor species turnover through time, changes in plant community composition were assessed indirectly by comparing morphotype and species diversity in different habitats, to check whether changes could be detected across habitat types. We first used Mantel correlation tests to evaluate whether the estimation of species turnover between plots was correlated with that found with morphotypes. Second, we investigated species, as well as morphotype turnover among the different habitats (i.e. types of margins). This was done using a non-parametric multivariate analysis of variance (PERMANOVA) with the FORTRAN program NPMANOVA (Anderson 2005). This method generates an F-ratio, the ratio of differences among habitat types 
on differences among plots within habitat type (Anderson 2001). Significance was determined from 100 random permutations with a significant level of $P=0.05$. Pairwise a posteriori comparisons were performed to quantify individual differences between pairs of habitats. Similar multivariate dispersion of points among groups was checked using FORTRAN program PERMDISP (Anderson 2004).

In the "multiple volunteers" experiment, the following analyses were performed in addition to those listed above. First, we compared the number of morphotypes found by each volunteer with the true number of species using Wilcoxon rank test. Second, we evaluated the "true" morphotype identification accuracy, which measures the proportion of morphotypes corresponding to a single taxonomically identified species. This measure was then compared to gross accuracy using a Wilcoxon rank test. Third, the F-ratio values obtained by non-parametric MANOVA were compared between botanists and volunteers, using Mann-Whitney rank test, to assess differences in accuracy of turnover revealed by species vs. morphotypes.

Non-metric multidimentional scaling (nMDS) was performed to provide a graphical representation of the differences among morphotype assemblages for each volunteer. This ordination method was also performed on species data and used as a reference point.

The robustness of the parataxonomic method was evaluated by two criteria: reproducibility (i.e. differences in morphotype identification among observers) and repeatability (i.e. difference in morphotype identification among replicate plots for each volunteer). This was studied as follows. To assess reproducibility, nonparametric MANOVA was performed on data collected by botanists and volunteers to test the observer effect on the variability of species and morphotypes assemblages. To assess repeatability, we first estimated the variability within observers via a 
measure of diversity between replicates within the same habitat type, assuming that a volunteer who tends to change the morphotype attributed to a given species among replicates will increase this measure. Apportionment of quadratic entropy (APQE), a method allowing hierarchical decomposition of diversity (Pavoine and Dolédec 2005) was used to decompose species or morphotype diversity into three hierarchical levels: among habitats types, among plots within habitat type, and within plot. Percentages obtained were compared between botanists and volunteers, using Mann-Whitney rank tests. Second, we computed, for each volunteer, the ratio of the total number of morphotypes in all plots to the mean number of morphotypes per plot, for each species detected more than once. This ratio was then averaged across all species for each volunteer and can be considered as a "repeatability index": even though volunteers might frequently split a species into several morphotypes within a plot, they should use the same morphotypes across plots if the method is repeatable, hence yielding a repeatability index close to 1 . We tested whether this index was significantly different from one using wilcoxon tests.

For all analyses involving comparisons among classes of contrasted sizes (habitat type in the "volunteer vs. botanist" experiment, and observer type in the "multiple-volunteer" experiment), a resampling procedure was performed to generate multiple samples of equal size. All analyses, except non-parametric MANOVA, were performed with the $\mathrm{R}$ statistical package version 2.4.1 for Windows ( $\mathrm{R}$ Development Core Team 2004) using the vegan library version 1.8-5 (Oksanen et al. 2007) and the ade4 library version 1.4-2 (Chessel et al. 2006).

\section{Results}


"Botanist vs. volunteer" experiment

In a comparison of the efficiency of an untrained volunteer and a skilled botanist, gross accuracy was $80.5 \%$. Species richness and morphotype richness per plot were highly significantly correlated (Spearman rho=0.87; $\mathrm{P}<0.01$ ) and not significantly different from each other. As expected, habitat type had a significant effect on both species and morphotype richness (non-parametric MANOVA, table 1), with higher species and morphotypes richness in road sides. However, species data were much more powerful than morphotype data to detect pairwise differences between habitats: all five habitat types exhibited significantly different species assemblages, whereas morphotypes revealed significant differences only between road side and two other habitats (footpath and grass strip, pair-wise a posteriori comparisons, table 1).

"Multiple-volunteer" experiment

When multiple volunteers were compared to botanists, the gross accuracy of morphotype identification (ranging between $61 \%$ and $90 \%$ ) was significantly higher than real accuracy (ranging between $55 \%$ and $70 \%$, see table 2 for detailed values). Lumping (i.e. attribution of several morphotypes to a single species) and splitting (attribution of a same morphotype to several species) occurred for all volunteers: the average frequency of correct identification (a single morphotype corresponds to a single species and vice versa) was $61 \%$, with $16 \%$ of lumping and $23 \%$ of splitting (figure 3). As before, the correlation between species and morphotype richness per plot was significant for all volunteers (table 2), but total morphotype richness per 
volunteer was significantly smaller than total species richness, as might be expected since different species may have similar morphotypes.

Significant species turnover among habitat was detected with both species and morphotypes, but with an observer-dependent accuracy, as suggested by significant Mantel correlation coefficients (measuring correlations between amonghabitat distances in botanists vs. volunteers), ranging between 0.46 and 0.77 . Both species and morphotype assemblages differed significantly among habitat types, as shown by non-parametric MANOVA (table 3). F-ratios obtained with botanist data were not significantly higher than those obtained with volunteers. Nevertheless, some differences in species assemblage among habitats remain undetected by four volunteers, as demonstrated by pairwise a posteriori comparison. Hence, information based on species more accurately revealed assemblage turn-over among habitat types. This result is supported by a visual assessment of patterns in the non-metric multidimensional scaling plot of data using Bray-Curtis distances, as shown in Figure 2.

In addition to the habitat effect on morphotype assemblage, there was a strong volunteer effect, as well as a significant interaction between volunteer and habitat type (non-parametric MANOVA, table 3). This shows that morphotype identification appeared to differ significantly among volunteers. In contrast, only habitat effect was found using botanist data (table 3), so that we can assume that there was no significant differences in species identification among botanists. For this reason, botanists were represented as a consensus (i.e. based on all species found by all botanists) in nMDS graphical representation (Figure 2). Finally, no significant differences were found between botanists and volunteers in the proportion of diversity between replicates within habitat type (APQE, Table 4; Kruskal-Wallis test; 
$P<0.05)$, whereas the repeatability index was much above $1(1.95 \pm 0.14$, Table 4$)$ indicating a lack of repeatability.

\section{Discussion}

In the context of large scale biodiversity monitoring, opening up to nonprofessional naturalists (Fore et al. 2001; Julliard and Jiguet 2002a; Stewart et al. 2006), the aim of this study was to explore the use of parataxonomy as a surrogate for species identification. In particular, we wanted to evaluate whether $\alpha$ and $\beta$ diversity, two essential indices in biodiversity monitoring schemes, were accurately measured using morphotypes instead of true species. We discuss the consequences of our findings for large scale biodiversity monitoring in the wider countryside.

\section{Estimation of $\alpha$ and $\beta$ diversity using morphotypes}

Morphotypes are widely used in taxonomy as a first step before determination (for example see the INBio project in Janzen, 2004) or in ecological studies to assess and survey species diversity, as in the present study (Basset et al. 2001). In the latter case, morphotypes are generally used with invertebrate taxa known to be speciesrich, and for which there often is limited taxonomic expertise, e.g. Araneae (Derraik et al. 2002), Diptera (Cranston and Hillman 1992), Coleoptera (Oliver and Beattie 1996a), Annelida (Oliver and Beattie 1993), and Formicidae (Pik et al. 1999). Nonetheless, as highlighted by Krell (2004) the real accuracy of morphotype identification often remains unknown, except in few studies which systematically compared morphotype identification vs. species determination (Oliver and Beattie 
1993; Oliver and Beattie 1996b; Derraik et al. 2002; Majka and Bondrup-Nielsen 2006). These studies demonstrated that the real accuracy can differ greatly among taxonomic groups, due to difficulties to separate different species on the basis of morphology. As an example, a low accuracy can be explained by intraspecific polymorphism in some groups (e.g. sexual or developmental differences leading to different colours or sizes in the same species), which leads to splitting or lumping species, whereas the diagnostic criteria remain cryptic for a non specialist (e.g. genitalia in Araneae).

Our results for vascular plants suggest an accurate estimation of species $\alpha$ diversity using morphotypes, as shown by the strong correlation between species and morphotype numbers in both experiments, which is consistent with earlier studies on invertebrate groups cited above (Oliver and Beattie 1993; Oliver and Beattie 1996b; Derraik et al. 2002; Majka and Bondrup-Nielsen 2006). Nevertheless, these gross correlations based only on the number of morphotypes must be considered cautiously, as suggested by significantly smaller real vs. gross accuracy. This is explained by the fact that splitting and lumping of species compensate each other in the gross accuracy value. Thus, the correlation between species number and morphotype number can be viewed as partly fortuitous, as already demonstrated on various invertebrates groups (Derraik et al. 2002; Barratt et al. 2003) and bryophytes (Oliver and Beattie 1993).

In all comparisons, species were successfully used to discriminate habitat types. In contrast, morphotypes appeared less sensitive than species to habitat differences, although they proved useful to detect the largest changes. This result is consistent with those found on spiders (Oliver and Beattie 1996b), but differs from results on other groups, such as ants (Pik et al. 1999) or beetles (Oliver and Beattie 
1996b; Majka and Bondrup-Nielsen 2006); in the two latter cases, morphotypes were sufficient to characterise $\beta$-diversity. We thus demonstrate that plant morphotypes, although useful for assessing species turnover in some situations, is not the most relevant approach due to a lower level of habitat discrimination than species.

Can parataxonomy be considered a scientific method?

The use of parataxonomy also questions the scientific criteria of our thought process. To our knowledge no study has ever tested repeatability (within observers) or reproducibility (across observers) in morphotype identification (but see Oliver and Beattie, 1993, who calculated the standard deviation of real accuracy among observers). In a recent review, Krell (2004) argued that parataxonomy cannot be considered a scientific activity due to limited reproducibility, revealed by large variation in gross accuracy for a given taxonomic group among studies, which, he hypothesized, was partly attributable to variation in morphotype identification among sorters. Our results in the 'multiple volunteers' experiment support Krell's concerns: the strong 'volunteer' effect demonstrates that we cannot assume reproducibility of morphotypes identification across observers. In addition, we also demonstrated that parataxonomy may not be repeatable. The differences among volunteers could not be detected with APQE measures (showing no significant differences between botanists and volunteers) but were obvious from the examination of repeatability indices: on average, volunteers used twice as many morphotypes to describe a species across replicate plots than within plots. The difference between APQE measures and repeatability indices may be explained by the fact that the former reflect only variation in morphotype identification in the same habitat whereas the 
later encompass all habitats. The only way to overcome reproducibility and repeatability problems is to use more detailed and constrained methods, such as dichotomous identification keys and collection of voucher specimens deposited in herbarium, which may be as difficult and time-consuming as true taxonomy.

However, other methods of inventory based on true taxonomy and generally considered scientific methods also exhibit limited reproducibility. Variation among botanists, although rarely tested, can be significant (Rich and Smith 1996; Rich 1998), inducing a pseudo-turn-over effect (Leps and Hadincova 1992; Rich and Woodruff 1992; Oredsson 2000; Kercher et al. 2003; Scott and Hallam 2003; Archaux et al. 2006). Hence, variation in species detectability and reproducibility across observers are significant constraints in all inventory methods. Nevertheless, as supported by our results on reproducibility and repeatability, we can assume that parataxonomy is more liable to such problems.

Parataxonomy should thus not be used in monitoring programs, unless its main limitation (lack of robustness) can be overcome. For example, computer assisted tools might help reducing the variability among observers. This can be done in two ways: (1) by providing databases with pictures of the different morphotypes, filled in by parataxonomists themselves, who can upload their own pictures to compare morphotypes with voucher specimens (Basset et al. 2000; Oliver et al. 2000), (2) by using computer-aided identification that allows automatic species identification on the basis of morphological characters (Gaston and O'Neill 2004). To our knowledge such approaches have been mainly developed with arthropods (Do et al. 1999; Arbuckle 2002; Watson et al. 2003) in response to the "taxonomic impediment" (Wheeler et al. 2004), but they could be used by amateurs to monitor plants in the near future, with the development of online databases (e.g. 
NatureGate® project, Åhlberg et al. 2006). Keeping in mind our results on accuracy, we can assume that, although these expensive methods are likely more reproducible than the one we used, they are also sensitive to the problem of intraspecific dimorphism, and must be implemented with caution, especially to monitor species turnover.

Can parataxonomic data be used for large scale monitoring of plants in common ecosystems?

Many examples of large scale biomonitoring programs in common ecosystems involve the work of hundreds of amateurs (coordinated by professionals) using standardized protocols, repeated over time (Stewart et al. 2006). These programs essentially consist of recording the presence and absence of species in a given area and mapping their distribution (Teder et al. 2007). Data collected can be used to assess how the abundance of species (estimated e.g. as the number of replicates in which a species has been recorded) changes between successive surveys (Smart et al. 2005), thus enabling the detection of local extinction or increase in frequency of a given species. Coupling such data with information on species characteristics (e.g. life history trait, habitat preferences, global distributions) and environmental data (e.g. habitat destruction, fragmentation, nutrient enrichment, climate changes) may help assessing some of the mechanisms responsible for biodiversity loss (Smart et al. 2003; Smart et al. 2005). For example, biotic homogenisation, i.e. the gradual replacement of specialist species by a few generalist species (McKinney and Lockwood 1999; Olden et al. 2004; Rooney et al. 2004) can be detected by repeated surveys (Smart et al. 2006). 
In comparison, the use of parataxonomic data in large scale monitoring can be a pitfall, if used without caution to address these issues. First, morphotypes are less accurate than species at detecting turnover among habitats. In addition, lack of reproducibility and repeatability in morphotype identification may artificially inflate differences among habitats or across time. Finally, species characteristics can not be incorporated in such monitoring programs, due to the impossibility of assigning a single code to a given species. Therefore, a large scale change, if detected, may not be easily interpreted due to the impossibility of assessing the direction and drivers of changes.

\section{Conclusion}

Parataxonomy can be viewed as a useful complement to taxonomic methods, enabling to quickly include the wider landscape matrix in global monitoring programs, where naturalist knowledge is often unavailable. This could allow the redeployment of skilled naturalists in « hotspots » (Oliver and Beattie 1997) where identification of rare species requires high levels of competence, and enable the multiplication of replicates at large scale, encompassing all habitat types (Oliver et al. 2000). Nevertheless, parataxonomy cannot be considered a sustainable solution to the loss of taxonomists. In developed countries, morphotypes must be viewed only as a temporary solution, providing incomplete data, which is always better than no data, while taxonomist skills are missing. But in the long term conservationists and ecologists will not be able to do without naturalist skills. Species are more than just a code number, without biological content (Goldstein 1997), they are essential knowledge to achieve the exploration of community processes and diversity. 


\section{Acknowledgments}

The authors thank Lorraine Bottin, Florence Devers, Margaret Evans, Anne-Sophie

Gadot, Isabelle Le Viol, Jean-Baptist Mihoub, and Audrey Muratet for their precious help in field experiments. We also acknowledge Tim Rich and one anonymous reviewer for helpful comments which greatly improved this manuscript. This study was funded by lle-de-France region.

\section{References}

Åhlberg M, Lehmuskallio E, Lehmuskallio J (2006) NatureGate®, concept mapping and CmapTools: Creating global networks of servers for improved learning about, in and for nature, ecosystems, biodiversity, and sustainable development. In: Canas A, Novak J (ed) Proceedings of the Second International Conference on Concept Mapping, San José, Costa Rica, 2006

Anderson MJ (2001) A new method for non-parametric multivariate analysis of variance. Austral Ecology 26:32-46

Anderson MJ (2004) PERMDISP: a FORTRAN computer program for permutational analysis of multivariate dispersions (for any two-factor ANOVA design) using permutation tests. Department of Statistics, University of Auckland, New Zealand.

Anderson MJ (2005) PERMANOVA: a FORTRAN computer program for permutational multivariate analysis of variance. Department of Statistics, University of Auckland, New Zealand.

Andrianandrasana HT, Randriamahefasoa J, Durbin J et al (2005) Participatory Ecological Monitoring of the Alaotra Wetlands in Madagascar. Biodiversity and Conservation 14:2757-2774

Arbuckle T (2002) Automatic identification of bees' species from images of their wings. In: World Scientific (ed) international workshop on systems, signals and image processing, Manchester, United-Kingdom, 2002

Archaux F, Gosselin F, Bergès L et al (2006) Effects of sampling time, species richness and observer on the exhaustiveness of plant censuses. 17:299-306

Barratt BIP, Derraik JGB, Rufaut CG et al (2003) Morphospecies as a substitute for Coleoptera species identification, and the value of experience in improving accuracy. Journal of the Royal Society of New Zealand 33:583-590

Basset Y, Charles E, Hammond DS et al (2001) Short-term effects of canopy openness on insect herbivores in a rain forest in Guyana. Journal of Applied Ecology 38:1045-1058 
Basset Y, Novotny V, Miller S et al (2000) Quantifying Biodiversity: Experience with Parataxonomists and Digital Photography in Papua New Guinea and Guyana. BioScience 50:899-908

Basset Y, Novotny V, Miller SE et al (2004) Conservation and biological monitoring of tropical forests: the role of parataxonomists. Journal of Applied Ecology 41:163-174

Bolger DT, Suarez AV, Crooks KR et al (2000) Arthropods in Urban Habitat Fragments in Southern California: Area, Age, and Edge Effects. Ecological Applications 10:1230-1248

Bray JR, Curtis JT (1957) An ordination of the upland forest communities of southern Wisconsin. Wisconsin. Ecol. Monographs 27:325-349

Cassey P, Blackburn TM (2006) Reproducibility and repeatability in ecology. BioScience 56:958-959

Chessel D, Dufour A-B, Dray S et al (2006) ade4: Analysis of Environmental Data : Exploratory and Euclidean method. Lyon, France

Clarke KR (1993) Non-parametric multivariate analyses of changes in community structure. Austral Ecology 18:117-143

Cranston P, Hillman T (1992) Rapid assessment of biodiversity using 'biological diversity technicians'. Australian Biologist 5:144-154

Derraik JGB, Closs GP, Dickinson KJM et al (2002) Arthropod Morphospecies versus Taxonomic Species: a Case Study with Araneae, Coleoptera, and Lepidoptera. Conservation Biology 16:1015-1023

Do MT, Harp JM, Norris KC (1999) A test of a pattern recognition system for identification of spiders. Bulletin of Entomological Research 89:217-224

Engel SM, Voshell JR (2002) Volunteer biological monitoring: Can it accurately assess the ecological condition of streams? American Entomologist 48:164177

Evans C, Abrams E, Reitsma R et al (2005) The Neighborhood Nestwatch Program: Participant Outcomes of a Citizen-Science Ecological Research Project. Conservation Biology 19:589-594

Firehock K, West J (1995) A Brief History of Volunteer Biological Water Monitoring Using Macroinvertebrates. Journal of the North American Benthological Society $14: 197-202$

Fischer J, Lindenmayer DB (2007) Landscape modification and habitat fragmentation: a synthesis. Global Ecology and Biogeography 16:265-280

Fore LS, Paulsen K, O'Laughlin K (2001) Assessing the performance of volunteers in monitoring streams. Freshwater Biology 46:109-123

Foster-Smith J, Evans SM (2003) The value of marine ecological data collected by volunteers. Biological Conservation 113:199-213

Fried G, Bombarde M, Delos M et al (2005) Evolution de la flore du maïs : Mauvaises herbes, ce qui a changé en 30 ans. Phytoma, la défense des végétaux 586:47-51

Gaston KJ, Fuller RA (2007) Commonness, population depletion and conservation biology. Trends in Ecology \& Evolution (in press)

Gaston KJ, May RM (1992) Taxonomy of taxonomists. Nature 356:281-282

Gaston KJ, O'Neill MA (2004) Automated species identification: why not? Philosophical Transactions of the Royal Society B: Biological Sciences 359:655-667 
Goldstein PZ (1997) How Many Things Are There? A Reply to Oliver and Beattie, Beattie and Oliver, Oliver and Beattie, and Oliver and Beattie. Conservation Biology 11:571-574

Holck MH (2007) Participatory forest monitoring: an assessment of the accuracy of simple cost-effective methods. Biodiversity and Conservation (in press):

Hopkins GW, Freckleton RP (2002) Declines in the numbers of amateur and professional taxonomists: implications for conservation. Animal Conservation 5:245-249

Janzen DH (2004) Setting up tropical biodiversity for conservation through nondamaging use: participation by parataxonomists. Journal of Applied Ecology 41:181-187

Julliard R, Jiguet $F$ (2002a) Un suivi intégré des populations d'oiseaux communs en France. Alauda 70:137-147

Julliard $R$, Jiguet $F(2002 b)$ Un suivi intégré des populations d'oiseaux communs en France. Alauda 70:137-147

Kercher SM, Frieswyk CB, Zedler JB (2003) Effects of sampling teams and estimation methods on the assessment of plant cover. Journal of Vegetation Science 14:899-906

Kerguélen M. 2003. Base de Données Nomenclaturales de la Flore de France (BDNFF) Version 3. Institut Français de la Biodiversité (ed).

. Cited 10 Nov 2007

Kerr JT, Sugar A, Packer L (2000) Indicator Taxa, Rapid Biodiversity Assessment, and Nestedness in an Endangered Ecosystem. Conservation Biology 14:17261734

Krell F-T (2004) Parataxonomy vs. taxonomy in biodiversity studies - pitfalls and applicability of "morphospecies" sorting. Biodiversity and Conservation 13:795812

Leps J, Hadincova V (1992) How Reliable Are Our Vegetation Analyses? Journal of Vegetation Science 3:119-124

Longino JT, Colwell RK (1997) Biodiversity Assessment Using Structured Inventory: Capturing the Ant Fauna of a Tropical Rain Forest. Ecological Applications 7:1263-1277

Lundmark C (2003) BioBlitz: Getting into Backyard Biodiversity. BioScience 53:329329

Majka CG, Bondrup-Nielsen S (2006) Parataxonomy: a test case using beetles. Animal Biodiversity and Conservation 29:149-156

McKinney ML (2006) Urbanization as a major cause of biotic homogenization. Biological Conservation 127:247-260

McKinney ML, Lockwood JL (1999) Biotic homogenization: a few winners replacing many losers in the next mass extinction. Trends in Ecology \& Evolution 14:450-453

Newson SE, Woodburn R, Noble DG et al (2005) Evaluating the Breeding Bird Survey for producing national population size and density estimates. Bird Study 52:42-54

Nielsen DL, Shiel RJ, Smith FJ (1998) Ecology versus taxonomy: is there a middle ground? Hydrobiologia 387:451-457

Noss RF (1996) The Naturalists Are Dying off. Conservation Biology 10:1-3

Oksanen J, Kindt R, Legendre P et al (2007) vegan: Community Ecology Package. 
Olden JD, Poff NL, Douglas MR et al (2004) Ecological and evolutionary consequences of biotic homogenization. Trends in Ecology \& Evolution 19:1824

Oliver I, Beattie AJ (1993) A Possible Method for the Rapid Assessment of Biodiversity. Conservation Biology 7:562-568

Oliver I, Beattie AJ (1996a) Designing a Cost-Effective Invertebrate Survey: A Test of Methods for Rapid Assessment of Biodiversity. Ecological Applications 6:594607

Oliver I, Beattie AJ (1996b) Invertebrate Morphospecies as Surrogates for Species: A Case Study. Conservation Biology 10:99-109

Oliver I, Beattie AJ (1997) Future Taxonomic Partnerships: Reply to Goldstein. Conservation Biology 11:575-576

Oliver I, Pik A, Britton D et al (2000) Virtual Biodiversity Assessment Systems. BioScience 50:441-450

Oredsson A (2000) Choice of surveyor is vital to the reliability of floristic change studies. Watsonia 23:287-291

Pavoine S, Dolédec S (2005) The apportionment of quadratic entropy: a useful alternative for partitioning diversity in ecological data. Environmental and Ecological Statistics 12:125-138

Penrose D, Call SM (1995) Volunteer Monitoring of Benthic Macroinvertebrates: Regulatory Biologists' Perspectives. Journal of the North American Benthological Society 14:203-209

Pereira HM, Cooper DH (2006) Towards the global monitoring of biodiversity change. Trends in Ecology \& Evolution 21:123-129

Perrings C, Jackson L, Bawa K et al (2006) Biodiversity in Agricultural Landscapes: Saving Natural Capital without Losing Interest. Conservation Biology 20:263264

Pik AJ, Oliver I, Beattie AJ (1999) Taxonomic sufficiency in ecological studies of terrestrial invertebrates. Austral Ecology 24:555-562

Pilgrim E, Hutchinson N. 2003. Bluebells for Britain - a report on the 2003 Bluebells for Britain survey. Plantlife and the BSBI, Salisbury, UK.

R Development Core Team (2004) R: A language and environment for statistical computing. R Foundation for Statistical Computing

Rich TCG (1998) Squaring the circles - bias in distribution maps. British Wildlife 9:213-219

Rich TCG (2006) Floristic changes in vascular plants in the British Isles: geographical and temporal variation in botanical activity 1836-1988. Botanical Journal of the Linnean Society 152:303-330

Rich TCG, Smith PA (1996) Botanical recording, distribution maps and species frequency. Watsonia 21:155-167

Rich TCG, Woodruff DS (1992) Recording bias in botanical survey. Watsonia 19:7395

Rivas JA (1997) Natural History: Hobby or Science? Conservation Biology 11:811812

Rooney TP, Wiegmann SM, Rogers DA et al (2004) Biotic Impoverishment and Homogenization in Unfragmented Forest Understory Communities. Conservation Biology 18:787-798

Scott WA, Hallam CJ (2003) Assessing species misidentification rates through quality assurance of vegetation monitoring. Plant Ecology 165:101-115 
Smart SM, Bunce RGH, Marrs R et al (2005) Large-scale changes in the abundance of common higher plant species across Britain between 1978, 1990 and 1998 as a consequence of human activity: Tests of hypothesised changes in trait representation. Biological Conservation 124:355-371

Smart SM, Clarke RT, van de Poll HM et al (2003) National-scale vegetation change across Britain; an analysis of sample-based surveillance data from the Countryside Surveys of 1990 and 1998. Journal of Environmental Management 67:239-254

Smart SM, Ken Thompson K, Marrs RH et al (2006) Biotic homogenization and changes in species diversity across human-modified ecosystems. Proceedings of the Royal Society B: Biological Sciences 273:2659-2665

Stewart K, Preston C, Ellis B. 2006. People and Plants: mapping the UK's wild flora. Plantlife and the BSBI, Salisbury, UK.

Stoate C, Boatman ND, Borralho RJ et al (2001) Ecological impacts of arable intensification in Europe. Journal of Environmental Management 63:337-365

Teder T, Moora M, Roosaluste E et al (2007) Monitoring of Biological Diversity: a Common-Ground Approach. Conservation Biology 21:313-317

Trumbull DJ, Bonney R, Bascom D et al (2000) Thinking scientifically during participation in a citizen-science project. Science Education 84:265-275

Tscharntke T, Klein AM, Kruess A et al (2005) Landscape perspectives on agricultural intensification and biodiversity - ecosystem service management. Ecology letters 8:857-874

Ward DF, Larivière MC (2004) Terrestrial invertebrate surveys and rapid biodiversity assessment in New Zealand: lessons from Australia. New Zealand Journal of Ecology 28:151-159

Watson AT, O'Neill MA, Kitching J (2003) Automated identification of live moths (Macrolepidoptera) using Digital Automated Identification SYstem (DAISY). Systematics and Biodiversity 1:287-300

Wheeler QD (1995) Systematics, the scientific basis for inventories of biodiversity. Biodiversity and Conservation 4:476-489

Wheeler QD, Raven PH, Wilson EO (2004) Taxonomy: impediment or expedient. Science 303:285

Whitehead P (1990) Systematics: An Endangered Species. Systematic Zoology 39:179-184

Whittaker RH (1972) Evolution and Measurement of Species Diversity. Taxon 21:213-251

Yoccoz NG, Nichols JD, Boulinier T (2001) Monitoring of biological diversity in space and time. Trends in Ecology \& Evolution 16:446-453 


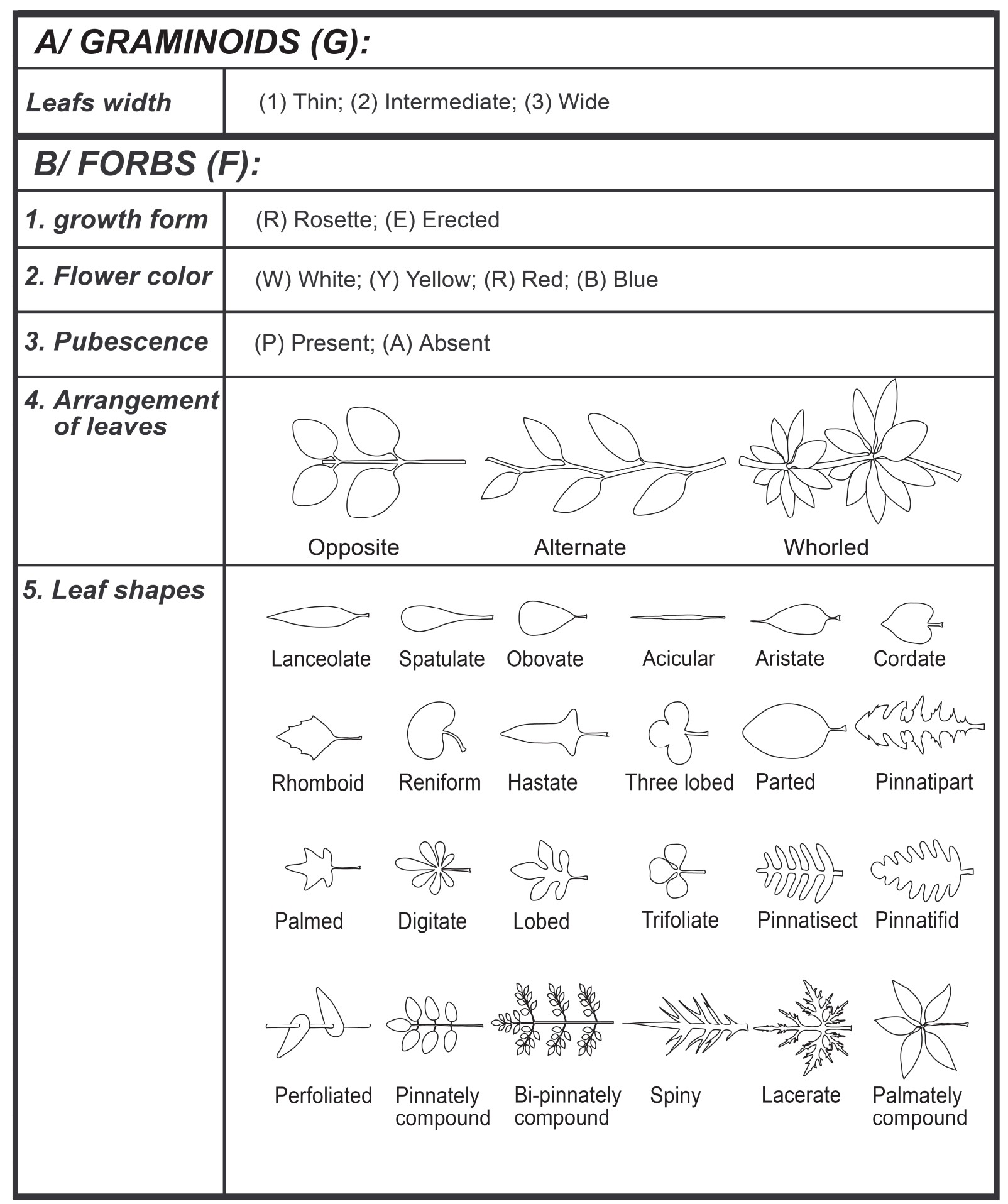

Figure 1: Criteria used in morphotype identification. Plants are first separated into graminoids (A) and forbs (B). A single character is considered for graminoids, whereas a list of five characters is provided for forbs. Each character is coded by a number corresponding to a given character state. A morphotype is defined as a combination of character states. 


\begin{tabular}{|c|c|c|}
\hline & \multicolumn{2}{|c|}{ F-ratio } \\
\hline & Species & Morphotype \\
\hline All habitats & 3.69 * & $2.27^{\star}$ \\
\hline \multicolumn{3}{|l|}{ Group comparison } \\
\hline footpath vs. wood edge & 1.71 * & 1.23 \\
\hline footpath vs. fallow land & 1.49 * & 1.04 \\
\hline footpath vs. grass strip & 2.00 * & 1.31 \\
\hline footpath $v s$. road side & 1.94 * & 1.86 * \\
\hline wood edge vs. fallow land & 1.75 * & 1.19 \\
\hline wood edge vs. grass strip & 2.32 * & 1.13 \\
\hline wood edge vs. road side & 1.75 * & 1.64 * \\
\hline fallow land vs. grass strip & 1.43 * & 1.24 \\
\hline fallow land vs. road side & 1.95 * & 2.26 * \\
\hline grass strip vs. road side & 2.57 * & 1.69 * \\
\hline
\end{tabular}

Table 1: Summary of non-parametric MANOVA in the 'botanist versus volunteer' experiment. Asterisks indicate significant differences between habitats $(P<0.05)$. 


\begin{tabular}{llllllllll}
\hline & Vol \# & Vol \# & Vol \# & Vol \# & Vol \# & Vol \# & Vol \# & Vol \# & \\
& 1 & 2 & 3 & 4 & 5 & 6 & 7 & 8 & Mean \\
\hline Morphotype richness & 39 & 57 & 51 & 54 & 53 & 53 & 45 & 56 & 51 \\
R-squared & 0.31 & 0.61 & 0.56 & 0.50 & 0.26 & 0.36 & 0.26 & 0.48 & 0.41 \\
Gross accuracy (\%) & 60.94 & 89.06 & 79.69 & 84.38 & 82.81 & 82.81 & 70.31 & 87.5 & 79.69 \\
Real accuracy (\%) & 63.33 & 60.71 & 69.7 & 59.09 & 56.07 & 64.76 & 54.74 & 60 & 61.05 \\
Lumping (\%) & 16.67 & 16.96 & 11.11 & 14.55 & 18.69 & 17.14 & 18.95 & 17.27 & 16.42 \\
Splitting (\%) & 20 & 22.32 & 19.19 & 26.36 & 25.23 & 18.1 & 26.32 & 22.73 & 22.53 \\
\hline
\end{tabular}

Table 2: Summary of diversity assessment by volunteers (Vol). A total of 64 species were sorted into various numbers of morphotypes (first line). The correlations between the number of species and the number of morphotypes per plot were all positive and significant; R-squared values are given on the second line. Gross accuracy is defined as $100-|(A-B) / A|$, where $A$ is the number of species and $B$ the number of morphotypes. Real accuracy corresponds to the proportion of correct identification. In the case of lumping, one or more morphotype is assigned to one species. Splitting correspond to the opposite case. 


\begin{tabular}{lrrrrr}
\hline \multicolumn{1}{c}{ Source } & $d f$ & \multicolumn{1}{c}{$S S$} & \multicolumn{1}{c}{$M S$} & \multicolumn{1}{c}{$F$} & \multicolumn{1}{c}{$P$} \\
\hline MORPHOTYPES & & & & & \\
observer & 7 & 105610.8679 & 15087.2668 & 8.0232 & $\mathbf{0 . 0 0 1 0}$ \\
habitat type & 3 & 96841.6164 & 32280.5388 & 6.2146 & $\mathbf{0 . 0 0 1 0}$ \\
$\begin{array}{l}\text { observer x habitat type } \\
\text { Residual }\end{array}$ & 21 & 109080.6259 & 5194.3155 & 2.7623 & $\mathbf{0 . 0 0 1 0}$ \\
& 128 & 240697.4089 & 1880.4485 & & \\
SPECIES & & & & & \\
observer & 2 & 1016.6018 & 508.3009 & 0.3226 & 0.9950 \\
habitat type & 3 & 100315.6419 & 33438.5473 & 71.9597 & $\mathbf{0 . 0 0 1 0}$ \\
observer x habitat type & 6 & 2788.1047 & 464.6841 & 0.2949 & 1.0000 \\
Residual & 48 & 75633.2211 & 1575.6921 & & \\
\hline
\end{tabular}

Table 3: Non-parametric MANOVA on Bray-Curtis distances for assemblage of morphotypes and species

\begin{tabular}{|c|c|c|c|c|c|c|c|c|c|c|c|}
\hline Diversity Source & Bot \# 1 & Bot \# 2 & Bot \# 3 & Vol \# 1 & Vol \# 2 & Vol \# 3 & Vol \# 4 & Vol \# 5 & Vol \# 6 & Vol \# 7 & Vol \# 8 \\
\hline $\begin{array}{l}\text { Between habitat } \\
\text { type }\end{array}$ & 3.7 & 4.3 & 4.5 & 3.4 & 3.2 & 4.3 & 2.7 & 3.2 & 5.3 & 3.3 & 3.4 \\
\hline $\begin{array}{l}\text { Between plot Within } \\
\text { habitat type }\end{array}$ & 5.5 & 6.7 & 7.5 & 6.8 & 7.2 & 6.7 & 6.9 & 7.6 & 9 & 9.1 & 6.4 \\
\hline Within plot & 90.9 & 89 & 88 & 89.8 & 89.5 & 89 & 90.4 & 89.2 & 85.7 & 87.7 & 90.2 \\
\hline Repeatability index & & & & 2.1 & 2 & 1.7 & 1.8 & 2.1 & 2 & 1.9 & 2 \\
\hline
\end{tabular}

Table 4: Repeatability of morphotype identification. The first three lines are results of the apportionment of quadratic entropy. Percentages correspond to decomposition of the total diversity found with species by botanists (Bot) and morphotypes by volunteers (Vol). Diversity among plots within habitat type was compared between botanists and volunteers as a first way to assess repeatability of morphotype identification. The repeatability index is the ratio of the total number of morphotypes to the mean number of morphotypes per plot, averaged over all species. 
Botanists (consensus)

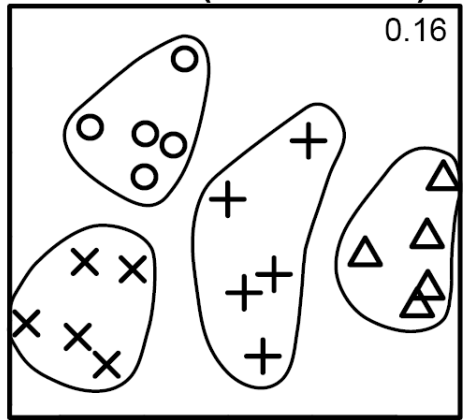

$\mathrm{F}=8.69$

Volunteer \# 3

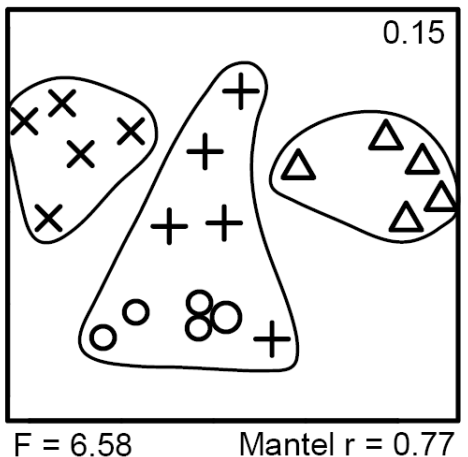

Volunteer \# 6

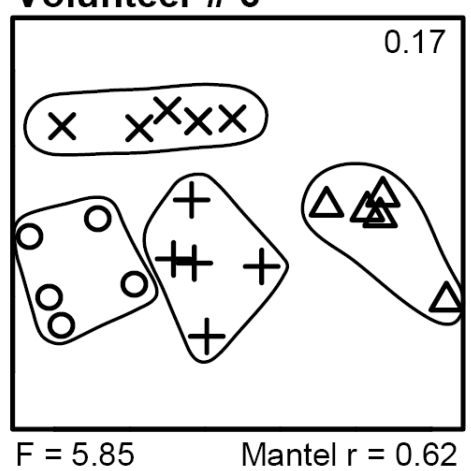

Volunteer \# 1

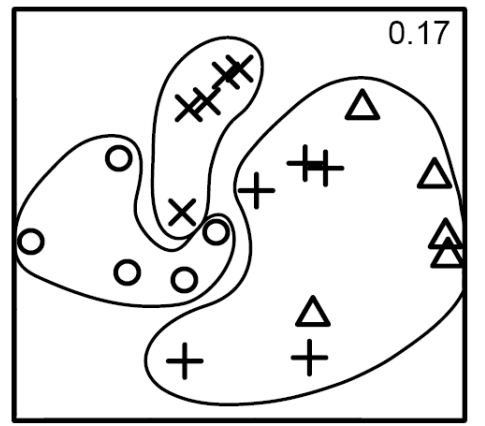

$F=4.99 \quad$ Mantel $r=0.56$

Volunteer \# 4

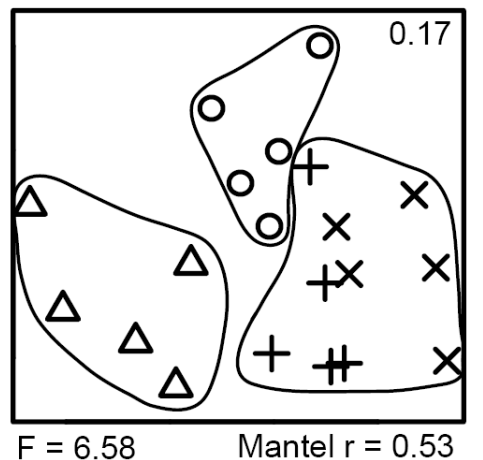

Volunteer \# 7

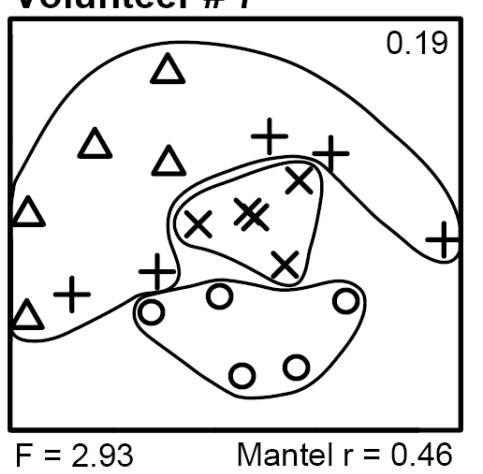

Volunteer \# 2

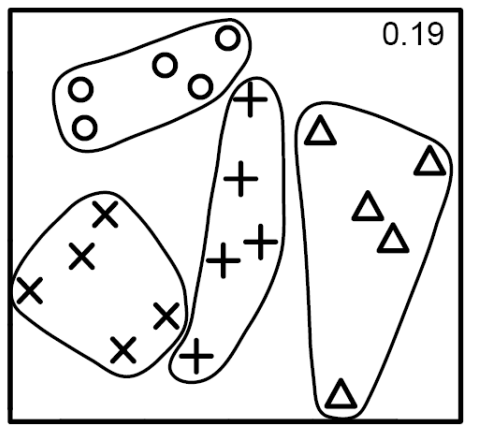

$\mathrm{F}=4.05 \quad$ Mantel $r=0.57$

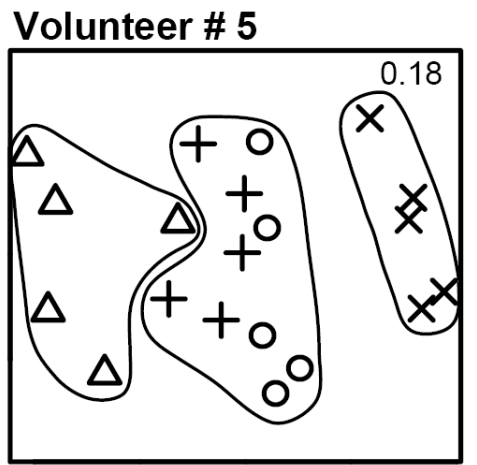

$\mathrm{F}=3.90 \quad$ Mantel $\mathrm{r}=0.56$

Volunteer \# 8

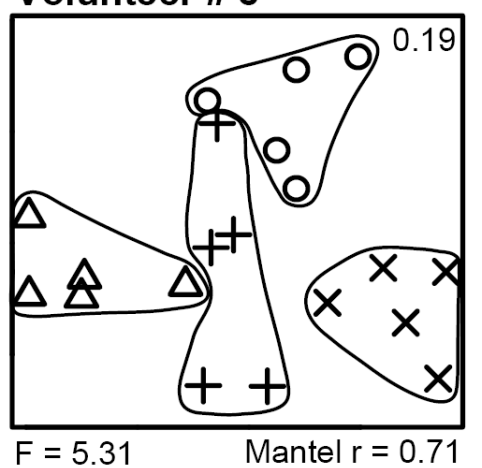

Figure 2: Non-Metric Multidimensional Scaling (NMDS) plot of data using Bray-Curtis distances. Each point represents a plot surveyed by a volunteer or by botanists.

Symbols indicate the four habitat types: (o), grass strip; $(\Delta)$, wood edge; $(x)$, hedge;

$(+)$, fallow land. Assuming that there are no significant differences among botanists, a consensus of species observed is presented. Stress values, measuring the disagreement between the inter-point distances on plot and these distances in the original distance matrix, are shown on the upper-right corner of each ordination scatter plot. PERMANOVA F-ratios and Mantel correlation coefficients between species assemblage and morphotype assemblage are displayed beneath each ordination. All are significant $(P<0.05)$. Habitat type considered by PERMANOVA pairwise comparisons to contain significantly different assemblages have been outlined by curves. 


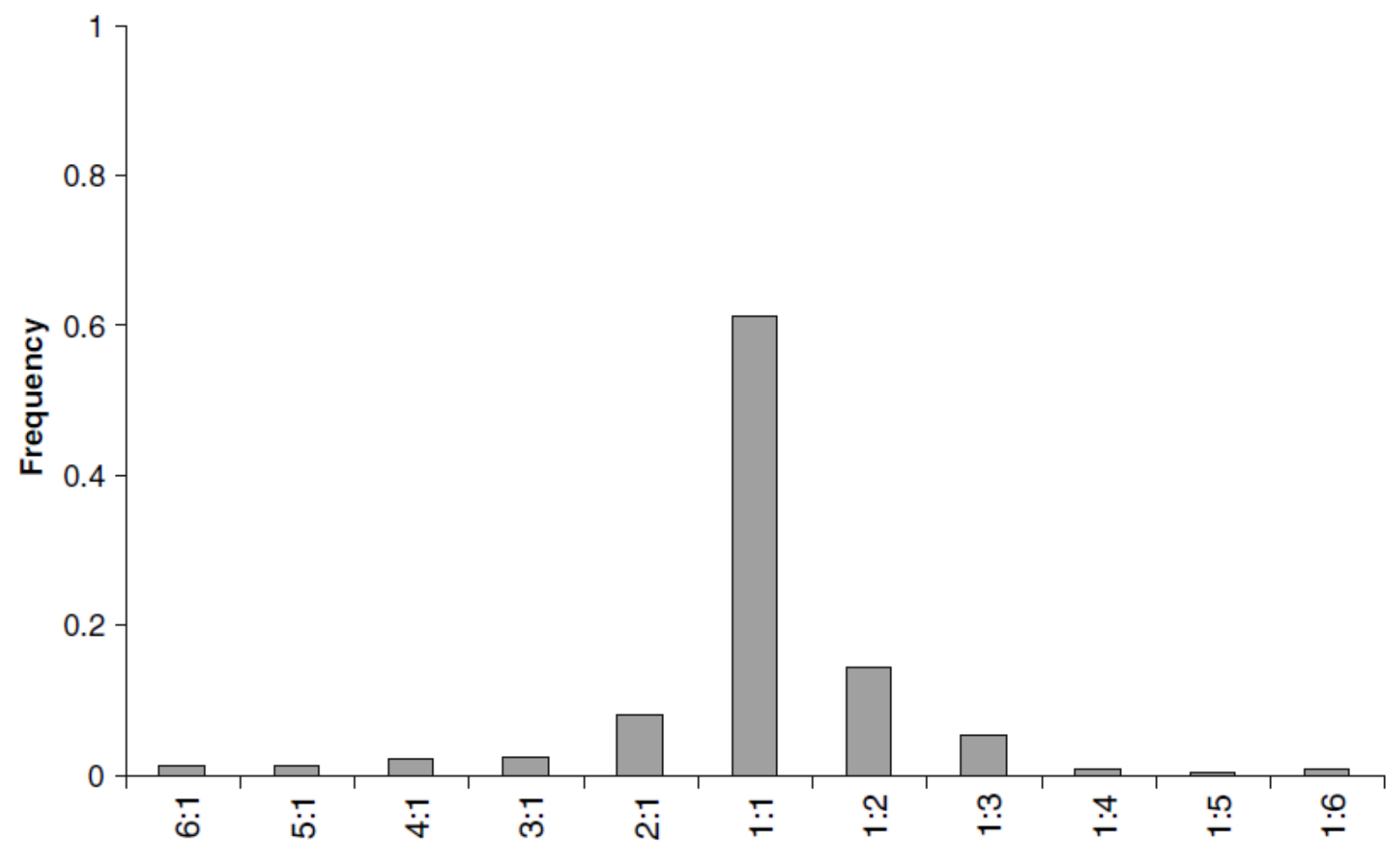

Ratio of species to morphotype

Figure 3: Mean ratio of species to morphotypes based on all volunteer data. The 1:1 class represents correct identifications. Classes to the right represent cases of splitting and those to the left represent cases of lumping. 